\title{
Évolution au champ de la croissance et élaboration du rendement d'un peuplement de blé d'hiver, en fonction des attaques de Rhizoctonia cerealis
}

\author{
P Lucas 1, N Cavelier 2, J Pierre 3, G Doussinault 3 \\ 1 INRA, Centre de recherche de Rennes, Station de pathologie végétale; \\ 2 SRIV; ${ }^{3}$ Station d'amélioration des plantes, BP 29, 35650 Le Rheu, France
}

(Reçu le 3 janvier 1990; accepté le 6 avril 1990)

\begin{abstract}
Résumé - Rhizoctonia cerealis, agent du rhizoctone des céréales, est susceptible d'attaquer la plante à tous les stades de son développement. Son extension récente est à mettre en relation avec les mesures prises pour limiter les attaques de piétin verse (Pseudocercosporella herpotrichoides), autre agent pathogène du pied des céréales. Par infestation artificielle des sols réalisée au moment du semis, il est possible de reproduire le rhizoctone, au champ, dès les stades jeunes de la plante. II a ainsi pu être montré, sur 4 années d'expérimentation, que des attaques précoces provoquent une destruction des jeunes plantes ou des premières talles en formation. De la mi-tallage jusqu'au début de la montaison, et bien que ce soit pendant cette période qu'on observe la plus grande fréquence de plantes atteintes, la maladie ne semble affecter ni la croissance, ni le développement des plantes. Pendant la montaison, la progression du champignon vers la tige, à partir des dernières gaines, même très attaquées, n'est pas aussi systématique que dans le cas du piétin verse. De plus, la nuisibilité du rhizoctone sur les dernières composantes du rendement (poids de 1000 grains et poids de grain par épi) n'a pu être démontrée qu'une seule année. Des 3 variétés de blé d'hiver étudiées, Arminda s'est révélée moins sensible au rhizoctone que Talent ou Roazon.
\end{abstract}

blé / rhizoctone / peuplement végétal / croissance / nuisibilité / rendement

Summary - Evolution, in the field, of the growth and of the yield components of a winter wheat crop, depending on attacks by Rhizoctonia cerealis. Rhizoctonia cerealis, causal agent of sharp-eyespot of cereals is more often observed in wheat crops. Its recent extension is linked to actions taken for controlling eyespot (Pseudocercosporella herpotrichoides). The symptoms of sharp-eyespot may be present at every growth stage of the plants and the aim of this study was to appreciate their incidence on the growth and on the yield components of a wheat crop. By artificial infestation of soils at sowing, we obtained a good development of the disease in the field, at early growth stages of the plant (figs 1,2). Through 4 years of experimentation, it has been shown that early attacks led to death of young plants (table I) and tillers of firth sheath (table II). The highest frequency of attacked plants was observed from mid tillering to the beginning of stem extension; however, the growth and the development of plants seemed to be unaffected (table III). During stem extension, the progress of the fungus from the last sheath to the stem did not occur as systematically as for the eyespot fungus (fig 3). Moreover, the effect of sharp-eyespot on the last yield components has been shown only one year (table IV). Among the 3 wheat varieties tested, Arminda appeared to be the least susceptible to sharp-eyespot, compared to Talent or Roazon (fig 1, table Vla). Even though, these results showed that yield losses due to sharp-eyespot were not so important, potential risks for the future are discussed due to the fact that controlling eyespot increases the parasitical activity of $\mathrm{R}$ cerealis.

wheat / sharp-eyespot / plant canopy / plant growth / yield component / yield loss 


\section{INTRODUCTION}

Bien que décrit pour la première fois en 1936 par Oort en Hollande, sous le nom de sharp eyespot disease, le rhizoctone des céréales a fait l'objet d'un nombre restreint d'études, comparé aux autres maladies du pied. C'est en 1937 que Sprague associe ces symptômes de taches ocellées à Rhizoctonia sp puis Rhizoctonia solani Kühn. En France, Foex (1941) signale des dégâts sur blés dus à un champignon à sclérote dont la description très détaillée laisse penser qu'il s'agit d'un Rhizoctonia. Sans donner de nom à cette affection, il conclut que la maladie qu'il observe présente des analogies avec l'affection de l'avoine et du blé décrite par Sprague dans l'Orégon. Glynne et Richtie (1943) observent dès 1935 des symptômes à la station de Rothamsted en Angleterre, puis en différents endroits de l'Angleterre et du Pays de Galles. La maladie considérée comme peu grave (en moyenne, $1 \%$ des tiges sont atteintes) est attribuée à $R$ solani.

Peu d'articles sont ensuite consacrés à cette maladie, les plus significatifs étant ceux de Pitt $(1964,1966)$ qui publie une synthèse d'observations sur le pouvoir pathogène des isolats de $R$ solani vis-à-vis des céréales et sur les conditions de développement de la maladie.

En 1977, Boerema et Verhoeven font de l'agent du rhizoctone une espèce nouvelle, $R$ hizoctonia cerealis Van der Hoeven. Cette nouvelle dénomination est très vite acceptée par l'ensemble des chercheurs qui se consacrent alors à l'étude de cette maladie : Reinecke et Fehrmann (1979), Lipps et Herr (1982), Lucas et Cavelier (1983), Hollins et al (1983). Ce regain d'intérêt s'explique par l'observation de plus en plus fréquente des symptômes de rhizoctone sur blé.

Ainsi, déjà en 1973, Rapilly et al signalent une aggravation de la maladie observable d'année en année, notamment en Bretagne. En 1979, Reinecke et Fehrmann montrent qu'il existe une corrélation négative entre développement au champ de $R$ cerealis et de Pseudocercosporella herpotrichoides (Fron) Deighton. Ils observent également qu'après application de fongicides contre le piétin verse, la fréquence d'attaques de rhizoctone, en moyenne, augmente. Les mêmes observations sont réalisées en France (Cavelier et al, 1985).

L'extension récente de cette maladie, liée à certaines pratiques culturales, a suscité quel- ques recherches sur sa réelle nuisibilité. Clarkson et Cook (1983) observent, en Angleterre et au Pays de Galles, que les lésions doivent concerner plus de la moitié de la circonférence de la tige pour occasionner des chutes de rendement, alors que Meunier (1984) en Belgique note que des attaques n'affectant pas la totalité de la circonférence de la tige ne sont pas préjudiciables. Mais toutes ces observations ne sont réalisées qu'à la récolte.

L'objet de l'étude présentée ici est de provoquer le développement du rhizoctone au champ par contamination artificielle et d'en apprécier la nuisibilité sur le développement et la croissance d'un peuplement végétal du semis à la maturité.

\section{MATÉRIEL ET MÉTHODES}

Toutes les observations sont réalisées au champ au cours de quatre campagnes successives entre 1983 et 1987.

\section{Matériel étudié}

La nuisibilité de $R$ cerealis est appréciée sur 3 variétés de blé d'hiver choisies sur la base de leur comportement vis-à-vis du rhizoctone (Lucas et Cavelier, 1983). Ainsi, Arminda est considérée comme peu sensible, contrairement à Talent et Roazon, cette variété étant par ailleurs choisie pour sa résistance vis-à-vis du piétin verse (Doussinault, 1970).

Ces variétés sont cultivées au champ (Domaine INRA du Rheu) dans des parcelles infestées ou non avec 2 isolats de $R$ cerealis, RC1 et RC6, susceptibles de provoquer des symptômes à tous les stades du développement de la plante.

L'inoculum est produit sur grains d'orge humidifiés par un volume égal en eau et stérilisés par 2 passages à l'autoclave $\left(1 \mathrm{~h}\right.$ à $\left.115^{\circ} \mathrm{C}\right)$, à $24 \mathrm{~h}$ d'intervalle. Après 3 semaines d'incubation à $20^{\circ} \mathrm{C}$, l'inoculum est séché à l'air pour être broyé et tamisé. Seules les particules ayant un diamètre compris entre 1 et $1,5 \mathrm{~mm}$ (= propagules) sont conservées pour infestation au champ.

\section{Dispositif expérimental}

Les semis de blé sont généralement réalisés dans la deuxième quinzaine d'octobre à raison de 330 grains/ $\mathrm{m}^{2}$. L'infestation des parcelles est effectuée en mélangeant aux semences de blé les propagules, de façon à apporter dans la raie de semis, l'équivalent de $1 \mathrm{~g}$ / $\mathrm{m}^{2}$. Une protection phytosanitaire est assurée contre les maladies du feuillage et le piétin verse par applica- 
tion d'un mélange de prochloraze $(300 \mathrm{~g} / \mathrm{l})$ et de carbendazime $(80 \mathrm{~g} / \mathrm{l})$ à raison de $1,5 \mathrm{l} / \mathrm{ha}$ au stade GS 31 de la culture (code décimal de Zadoks et al, 1974).

Le dispositif adopté est un split plot à 4 blocs, les grandes parcelles correspondant aux infestations par chacun des isolats et au témoin non infesté, les parcelles élémentaires $\left(12 \mathrm{~m}^{2}\right)$ aux variétés.

\section{Notations}

Les plantes sont prélevées au champ sur une longueur de rang de $10 \mathrm{~cm}$, à raison de 5 prises $(5 \times$ $10 \mathrm{~cm}$ ) par parcelle élémentaire. Sur ces plantes, sont notés, pour chaque placette et en fonction des stades de prélèvement suivants :

- mi-tallage ( $4^{e}$ ou $5^{e}$ talle en formation) : nombre de plantes, séquence de tallage des plantes;

- début montaison : nombre de plantes et de tiges, quantité de matière sèche aérienne produite, fréquence du rhizoctone et du piétin verse;

- floraison : nombre de plantes et d'épis, quantité de matière sèche aérienne produite, fréquence et intensité du piétin verse et du rhizoctone.

A maturité, le rendement parcellaire est mesuré. De plus, du fait de la présence fréquente de piétin verse dans nos parcelles, mais aussi de contaminations naturelles de rhizoctone dans celles non infestées artificiellement, l'effet du rhizoctone sur les composantes du rendement déterminées en fin de cycle a été mesuré à partir de lots de plantes obtenus comme suit : pour chaque parcelle, les plantes d'un rang $(4,50 \mathrm{~m})$ sont arrachées et triées en :

- plantes indemnes de rhizoctone et ne présentant aucune autre affection du pied (piétin échaudage ou piétin verse);

- plantes atteintes seulement de rhizoctone (au moins $25 \%$ de la tige nécrosée sur sa section).

Sur chacun de ces lots sont mesurés le poids de grains par épi et le poids de 1000 grains.

\section{RÉSULTATS}

\section{Évolution du rhizoctone au champ}

D'une manière générale, la méthode d'infestation utilisée permet une reproduction des symptômes tout à fait satisfaisante comme le montrent les résultats de l'année 1986-1987 pris à titre d'exemple (fig 1). En effet, dès la notation réalisée courant tallage (en mars), entre 55 et $65 \%$ des plantes des variétés Talent et Roazon sont attaquées. Par contre, si à ce stade le niveau d'attaque reste très faible dans les parcelles non infestées artificiellement, l'examen de la figure montre que, par la suite, la fréquence de plantes atteintes et le pourcentage de section nécrosée ne sont pas significativement différents $(P=$ 0,05 ), que ces parcelles soient ou non artificiellement infestées par $R$ cerealis (fig 1). Nous ne disposons en effet d'aucun moyen réellement efficace pour maintenir une parcelle indemne ou pour le moins à un niveau très faible de rhizoctone. Même si certaines années, la différence entre parcelle infestée et parcelle non infestée est plus importante, notamment pour la notation au stade floraison (exemple illustré dans la figure 2), nous avons été amenés à mesurer les dernières composantes du rendement sur plantes individuelles triées selon leur niveau d'attaque.

\section{Évolution du piétin verse et du rhizoctone au champ}

L'évolution du pourcentage de plantes atteintes par ces 2 maladies, illustrée par la figure 3 , montre 2 différences fondamentales :

- la progression non systématique du champignon à partir de la dernière gaine, jusqu'à la tige, dans le cas du rhizoctone;

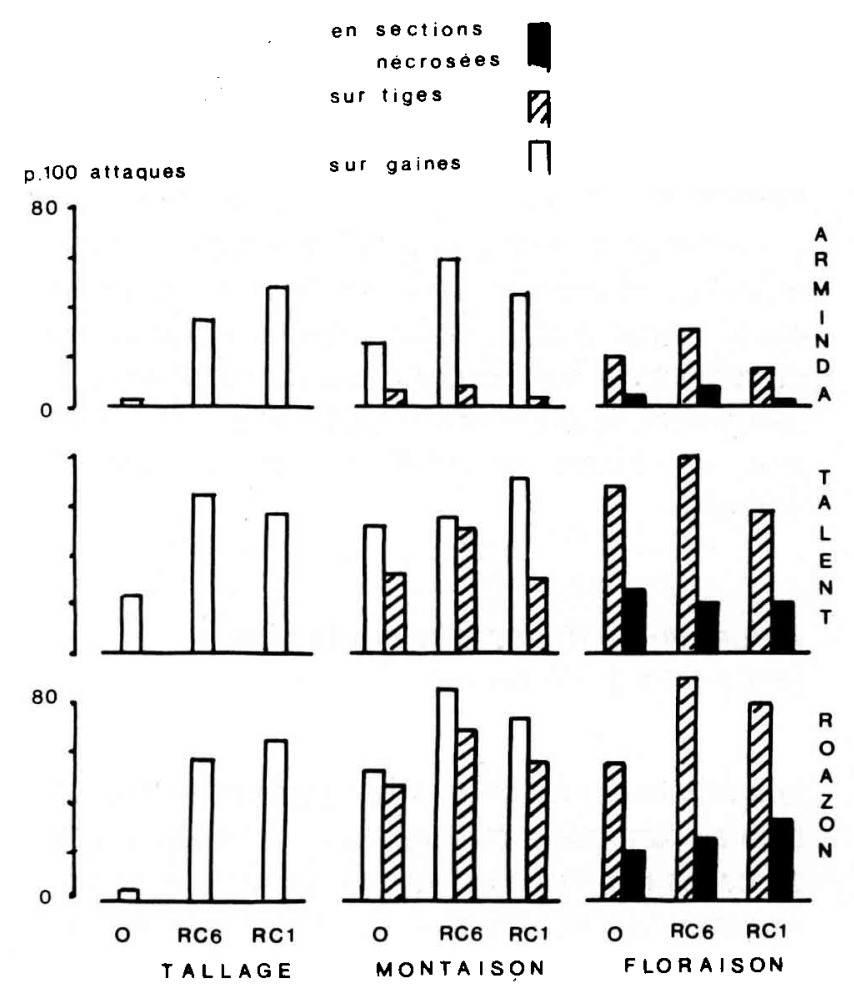

Fig 1. Évolution du pourcentage de plantes (tallage) ou de tiges et gaines (début montaison, fin floraison) atteintes de rhizoctone pour 3 variétés de blé cultivées en parcelles infestées artificiellement (RC6, RC1) ou non (O) avec $R$ cerealis. Campagne 1986-1987. 

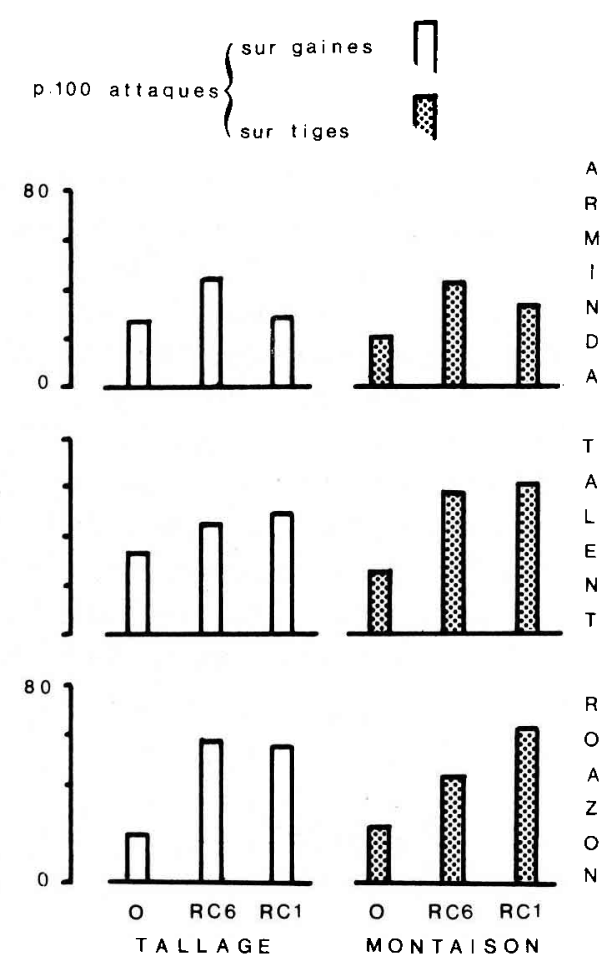

Fig 2. Évolution du pourcentage de tiges et gaines atteintes de rhizoctone pour 3 variétés de blé cultivées en parcelles infestées artificiellement (RC6, RC1), ou non (O) avec $R$ cerealis. Campagne 1985-1986.

- le caractère dominant du piétin verse, en l'absence de traitement fongicide contre celui-ci. En fin de végétation du blé, on observe même une diminution des symptômes à faciès rhizoctone due à leur colonisation progressive par $P$ herpotrichoides.

La figure 4 illustre la relation entre ces deux maladies et montre la nécessité là aussi d'une étude plante à plante pour mesurer la nuisibilité du rhizoctone sur les dernières composantes du rendement, la pression de piétin verse étant toujours supérieure en parcelle non infestée avec $R$ cerealis.

\section{Influence du rhizoctone sur la phase levée-tallage du blé}

Les notations réalisées à la mi-tallage $\left(4^{\mathrm{e}}\right.$ ou $5^{\mathrm{e}}$ talle en formation) montrent que l'infestation des parcelles par l'une ou l'autre souche de $R$ cerealis entraîne une réduction du nombre de plantes sur le rang. L'importance de cette réduction est très variable suivant les années et les variétés (tableau I), jusqu'à être nulle en 1984-1985. II est à signaler toutefois que le semis a été, pour cette campagne, réalisé très tardivement (début

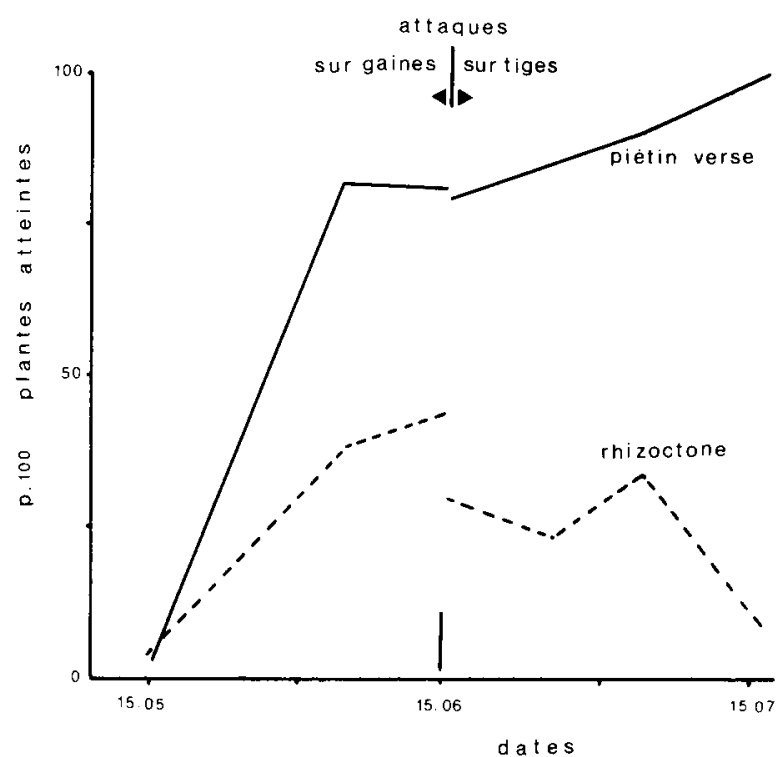

Fig 3. Évolution dans une même parcelle des attaques de piétin verse et de rhizoctone $(\%$ de plantes atteintes sur gaines, puis sur tiges). Le Rheu, 1985.

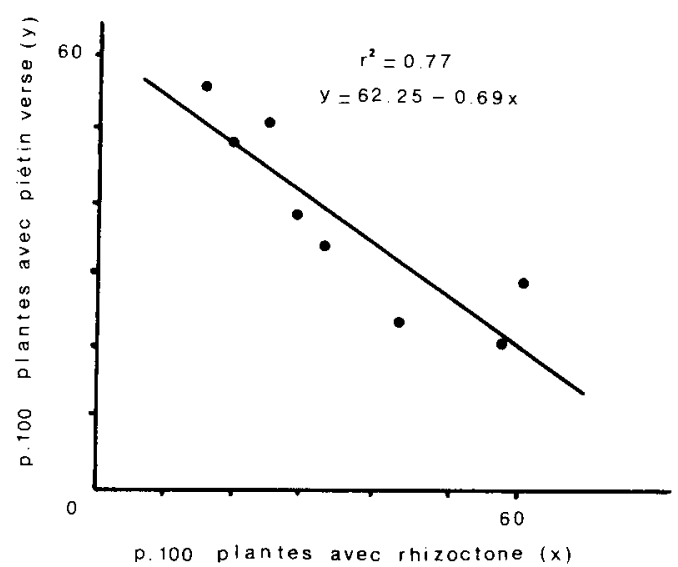

Fig 4. Relation entre les pourcentages respectifs de plantes atteintes sur tiges de rhizoctone et (ou) de piétin verse dans 8 parcelles du Rheu (1985). Chaque point représente les valeurs obtenues sur un échantillon par parcelle, de 4 prélèvements de 50 plantes.

décembre) par rapport aux campagnes précédente et suivante (mi-octobre) et que les conditions hivernales n'ont sans doute pas permis le développement de la maladie à partir de l'inoculum introduit.

Ces attaques précoces peuvent également nuire à la formation de la première talle comme le montrent certains résultats du tableau II. C'est surtout la variété Talent qui est affectée. II semble que pour la variété Roazon et pour Arminda (en 1985-1986) d'autres facteurs limitants aient influé sur la formation de cette première talle si l'on considère les valeurs faibles obtenues dans les parcelles non infestées. Néanmoins, en 1986-1987, Arminda, dont $86 \%$ des 
Tableau I. Diminution (en \%) de la densité de peuplement de plantes liée à l'infestation au semis par 2 isolats de $R$ cerealis (RC6 et RC1, notation mi-tallage). NC : Non Calculé.

\begin{tabular}{llccc}
\hline Années & Variétés & \multicolumn{3}{c}{ Inoculum apporté } \\
& & 0 & $R C 6$ & $R C 1$ \\
& & & & \\
\hline \multirow{3}{*}{$1983-1984$} & Arminda & 0 & 12 & 13,5 \\
& Talent & 0 & 12 & 20,5 \\
& Roazon & NC & NC & NC \\
& & & & \\
$1984-1985$ & Pas de différence (semis très tardif) \\
& & & & \\
$1985-1986$ & Arminda & 0 & 0 & 3 \\
& Talent & 0 & 9 & 9 \\
& Roazon & 0 & 8 & 16 \\
\hline
\end{tabular}

plantes présentent une talle de première feuille dans ces parcelles, paraît moins affectée que Talent par l'infestation, ce qui correspond aussi à un moins grand nombre de plantes de la variété Arminda atteintes à ce stade (voir la figure 1).

\section{Influence du rhizoctone sur la quantité de matière sèche formée au stade début montaison}

Pour chacune des expérimentations, la quantité de matière sèche aérienne produite par unité de longueur de rang est identique, quelle que soit

Tableau II.Pourcentage de plantes présentant une talle

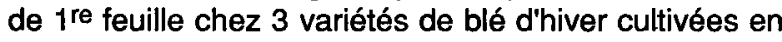
parcelles infestées ou non avec $R$ cerealis (isolats RC6 et $\mathrm{RC} 1$, notation mi-tallage).

\begin{tabular}{|c|c|c|c|c|}
\hline \multirow[t]{2}{*}{ Années } & \multirow[t]{2}{*}{ Variétés } & \multicolumn{3}{|c|}{ Inoculum apporté } \\
\hline & & 0 & RC6 & $R C 1$ \\
\hline $1985-1986$ & $\begin{array}{l}\text { Arminda } \\
\text { Talent } \\
\text { Roazon }\end{array}$ & $\begin{array}{l}66,0 \\
80,1 \\
53,1\end{array}$ & $\begin{array}{l}63,5 \\
58,5^{\star} \\
56,7\end{array}$ & $\begin{array}{l}60,9 \\
61,0^{\star} \\
41,5\end{array}$ \\
\hline $1986-1987$ & $\begin{array}{l}\text { Arminda } \\
\text { Talent } \\
\text { Roazon }\end{array}$ & $\begin{array}{l}85,3 \\
87,2 \\
56,5\end{array}$ & $\begin{array}{l}82,3 \\
70,7 \\
62,9\end{array}$ & $\begin{array}{l}75,0 \\
61,5 \\
63,9\end{array}$ \\
\hline
\end{tabular}

- Valeurs significativement différentes de la valeur obtenue pour la même variété en parcelle non infestée $(P=0,05)$. l'infestation, comme l'illustrent les résultats des campagnes 1985-1986 et 1986-1987 (tableau III) et ce, malgré les différences de densité de peuplement constatées.

\section{Influence des attaques du rhizoctone sur les composantes du rendement déterminées en fin de cycle}

\section{Résultats 1982-1983}

La première expérimentation destinée à évaluer la nuisibilité du rhizoctone sur une culture de blé a été mise en place à l'automne 1982 et ne concernait que la variété Roazon. Les observations réalisées l'ont été sur les dernières composantes du rendement à partir d'une étude plante à plante telle que décrite précédemment. Les résultats sont regroupés dans le tableau IV.

Ainsi, des attaques de rhizoctone, au cours de cette campagne, ont entraîné, chez la variété Roazon, une diminution de la productivité des épis de $12 \%$, le poids moyen de 1000 grains étant lui-même réduit de $5,5 \%$.

\section{Résultats 1984-1987}

\section{Peuplement épis}

Les observations au stade floraison du blé révèlent un effet variété, Talent ayant un peuplement épi supérieur à ceux de Roazon et Arminda. Elles ne révèlent, par contre, aucune différence entre les peuplements épis, que les parcelles soient infestées ou non par $R$ cerealis (tableau V).

Tableau III. Quantité de matière sèche formée par plante ou par unité de longueur sur le rang, toutes variétés confondues (Arminda, Talent, Roazon) en fonction de l'infestation des parcelles avec $R$ cerealis (notation début montaison). Les valeurs ne sont pas significativement différentes pour $P=0,05$.

\begin{tabular}{lccccccc}
\hline \multicolumn{1}{c}{$\begin{array}{c}\text { Quantité de matière sèche produite (cg) } \\
\text { par plante }\end{array}$} & \multicolumn{1}{c}{ pour $10 \mathrm{~cm}$ de rang } \\
Inoculum & 0 & $R C 6$ & $R C 1$ & 0 & $R C 6$ & $R C 1$ \\
& & & & & & \\
\hline Années & & & & & & & \\
$1985-1986$ & 305 & 327 & 340 & 1658 & 1479 & 1536 \\
$1986-1987$ & 326 & 331 & 367 & 1906 & 1906 & 1898 \\
\hline
\end{tabular}


Tableau IV. Influence des attaques de rhizoctone sur la formation des grains de la variété Roazon (campagne 1982-1983). Les valeurs présentées constituent les moyennes obtenues sur 8 échantillons (plantes arrachées sur un rang de $4,50 \mathrm{~m}$ dans 8 parcelles). Les valeurs d'une même colonne suivies de lettres différentes sont significativement différentes pour $P=0,05$.

\begin{tabular}{lccc}
\hline & $\begin{array}{c}\text { Nombre } \\
\text { moyen } \\
\text { de tiges par } \\
\text { échantillon }\end{array}$ & $\begin{array}{c}\text { Poids } \\
\text { moyen } \\
\text { grains/épi } \\
(g)\end{array}$ & $\begin{array}{c}\text { Poids } \\
\text { moyen }\end{array}$ \\
\hline $\begin{array}{l}\text { Tiges saines } \\
\text { Tiges avec } \\
\text { rhizoctone }\end{array}$ & 382 & $1,55 \mathrm{~b}$ & $43,2 \mathrm{~b}$ \\
$\begin{array}{c}\text { Pertes liées aux } \\
\text { attaques (\%) }\end{array}$ & 64 & $1,36 \mathrm{a}$ & $40,8 \mathrm{a}$ \\
\end{tabular}

\section{Poids de grains par épi et poids de 1000 grains}

Les résultats obtenus en 1984, 1986 et 1987 vont dans le même sens que ceux présentés à titre d'illustration dans le tableau VI et qui correspondent aux observations de juillet 1985. On peut constater qu'il n'apparaît aucune différence significative sur les variables poids de grains/épi (tableau Vlb) et poids de 1000 grains (tableau VIc) en fonction de l'attaque du rhizoctone.

Tableau V. Nombre d'épis par placette prélevée (10 cm sur le rang) pour 3 variétés de blé semées en parcelles infestées artificiellement (RC6, RC1) ou non infestées (0) avec $R$ cerealis. Les valeurs moyennes suivies d'une même lettre ne sont pas significativement différentes pour $P=0,05$.

\begin{tabular}{|c|c|c|c|c|c|}
\hline \multirow[t]{2}{*}{ Années } & \multirow[t]{2}{*}{ Variétés } & \multicolumn{3}{|c|}{ Inoculum apporté } & \multirow[t]{2}{*}{ Moyenne } \\
\hline & & 0 & $R C 6$ & $R C 1$ & \\
\hline $\begin{array}{l}1984- \\
1985\end{array}$ & $\begin{array}{l}\text { Arminda } \\
\text { Talent } \\
\text { Roazon } \\
\text { Moyenne }\end{array}$ & $\begin{array}{l}18,0 \\
22,2 \\
16,6 \\
18,9 a\end{array}$ & $\begin{array}{l}19,4 \\
19,6 \\
15,0 \\
18,0 \mathrm{a}\end{array}$ & $\begin{array}{l}18,4 \\
21,2 \\
13,4 \\
17,7 a\end{array}$ & $\begin{array}{l}18,6 a b \\
21,0 a \\
15 b\end{array}$ \\
\hline $\begin{array}{l}1986- \\
1987\end{array}$ & $\begin{array}{l}\text { Arminda } \\
\text { Talent } \\
\text { Roazon } \\
\text { Moyenne }\end{array}$ & $\begin{array}{l}16,2 \\
20,4 \\
14,7 \\
17,1 a\end{array}$ & $\begin{array}{l}14,7 \\
17,8 \\
14,2 \\
15,6 a\end{array}$ & $\begin{array}{l}15,4 \\
19,5 \\
13,2 \\
16,0 a\end{array}$ & $\begin{array}{l}15,4 b \\
19,2 a \\
14,1 b\end{array}$ \\
\hline
\end{tabular}

Tableau VI. Incidence, sur les dernières composantes du rendement, des attaques de rhizoctone sur la tige (au moins $25 \%$ de la section de tige nécrosée). Aucune différence n'apparaît significative pour $P=$ 0,05. A : effectifs moyens des lots analysés de tiges saines et attaquées. $B$ : poids moyen de grains par épi $(\mathrm{g})$ des 3 variétés présentant ou non des attaques de rhizoctone. $C$ : poids de 1000 grains obtenus pour les 3 variétés, à partir d'épis portés par des tiges saines ou atteintes de rhizoctone dans différentes parcelles artificiellement infestées ou non. ND : non déterminé, lots inférieurs à 500 grains.

\begin{tabular}{llrrrr} 
Variétés & Tiges & \multicolumn{3}{c}{ Inoculum apporté } & Moyenne \\
& & 0 & $R C 6$ & $R C 1$ & \\
& & & & & \\
\hline \multirow{4}{*}{ Arminda } & saines & 310 & 394 & 475 & 393 \\
& attaquées & 26 & 113 & 23 & 54 \\
\multirow{4}{*}{ Talent } & saines & 143 & 127 & 129 & 133 \\
& attaquées & 57 & 136 & 138 & 110 \\
& & & & & \\
Roazon & saines & 167 & 105 & 113 & 128 \\
& attaquées & 33 & 80 & 105 & 73
\end{tabular}

A

\begin{tabular}{|c|c|c|c|c|}
\hline \multirow[t]{2}{*}{ Variétés } & \multirow[t]{2}{*}{ Tiges } & \multicolumn{3}{|c|}{ Inoculum apporté } \\
\hline & & 0 & $R C 6$ & $R C 1$ \\
\hline Arminda & $\begin{array}{l}\text { saines } \\
\text { attaquées }\end{array}$ & $\begin{array}{l}2,2 \\
1,9\end{array}$ & $\begin{array}{l}2,1 \\
2,3\end{array}$ & $\begin{array}{l}2,1 \\
1,9\end{array}$ \\
\hline Talent & $\begin{array}{l}\text { saines } \\
\text { attaquées }\end{array}$ & $\begin{array}{l}1,3 \\
1,3\end{array}$ & $\begin{array}{l}1,5 \\
1,5\end{array}$ & $\begin{array}{l}1,5 \\
1,5\end{array}$ \\
\hline Roazon & $\begin{array}{l}\text { saines } \\
\text { attaquées }\end{array}$ & $\begin{array}{l}2,0 \\
2,0\end{array}$ & $\begin{array}{l}2,0 \\
2,2\end{array}$ & $\begin{array}{l}2,0 \\
2,1\end{array}$ \\
\hline \multirow[t]{2}{*}{ Variétés } & Tiges & \multicolumn{3}{|c|}{ Inoculum introduit } \\
\hline & & 0 & $R C 6$ & $R C 1$ \\
\hline Arminda & $\begin{array}{l}\text { saines } \\
\text { attaquées }\end{array}$ & $\begin{array}{l}46,9 \\
\text { ND }\end{array}$ & $\begin{array}{l}47,1 \\
45\end{array}$ & $\begin{array}{l}47,8 \\
\text { ND }\end{array}$ \\
\hline Talent & $\begin{array}{l}\text { saines } \\
\text { attaquées }\end{array}$ & $\begin{array}{l}41,4 \\
41,1\end{array}$ & $\begin{array}{l}41,7 \\
40,7\end{array}$ & $\begin{array}{l}41,6 \\
42,7\end{array}$ \\
\hline Roazon & $\begin{array}{l}\text { saines } \\
\text { attaquées }\end{array}$ & $\begin{array}{l}55,2 \\
56,2\end{array}$ & $\begin{array}{l}55,2 \\
55,3\end{array}$ & $\begin{array}{l}55,2 \\
56,4\end{array}$ \\
\hline
\end{tabular}


Seules des différences variétales peuvent être notées quant au poids de 1000 grains. Notons également que pour la variété Arminda, il ne nous a pas été possible de mesurer rigoureusement le poids de 1000 grains obtenus à partir de brins attaqués, l'échantillonnage étant trop faible. Ceci confirme chez Arminda la moindre sensibilité vis-à-vis du rhizoctone déjà observée (tableau Vla).

\section{DISCUSSION ET CONCLUSION}

Une infestation artificielle des sols au moment du semis permet donc de reproduire la maladie du rhizoctone sur un peuplement de blé au stade précoce. Les premières attaques apparaissent très tôt et se traduisent par la destruction de jeunes plantes. Elles nuisent également, dans quelques cas, à la formation de la talle de la jre feuille.

Pour la suite des observations, une des principales difficultés a été le maintien d'un état sanitaire convenable dans les parcelles non infestées artificiellement par $R$ cerealis et devant permettre une évolution optimale du peuplement végétal.

Les différences de niveau de maladie entre parcelles infestées ou non, observées certaines années, montrent qu'au début de la montaison, les quantités de matière sèche formées dans ces 2 situations, sont semblables. Pourtant, c'est à ce stade que sont enregistrées les plus fortes attaques de rhizoctone en terme de pourcentage de plantes atteintes. En ce sens, on doit considérer que si ces infections sont importantes d'un point de vue épidémiologique en maintenant une source d'inoculum susceptible d'infecter la tige, elles ne nuisent pas à la croissance ultérieure des plantes. II semble même y avoir au niveau de la plante individuelle une meilleure croissance en parcelle infestée (mais les différences ne sont pas significatives pour $P=0,05$ ), compensant la plus faible densité de peuplement.

Deux événements mal contrôlés ont perturbé notre étude comparative de l'évolution de peuplements de blé subissant des pressions différentes de rhizoctone. Le premier, lié à l'impossibilité de juguler une infestation naturelle dans les parcelles non infestées artificiellement, nous a privé de notre peuplement de référence sain, ou pour le moins peu attaqué en rhizoctone, comme ce fut plus particulièrement le cas en 1986-1987. Le second est lié au fait que le piétin verse, même en présence de traitements fongicides en végéta- tion, a un développement plus important en parcelles non contaminées avec $R$ cerealis. Ce biais est relativement gênant compte tenu de la nuisibilité du piétin verse antérieurement démontrée par Ponchet (1959) et Doussinault (1973). En effet, si l'incidence du piétin verse apparaît peu importante sur le peuplement épi, des attaques même faibles sur tige (moins de $10 \%$ de surface nécrosée) entraînent des diminutions du poids de 1000 grains et du nombre de grains formés.

Dans nos conditions d'expérimentation, nous n'avons pas relevé d'incidences des attaques de rhizoctone sur le peuplement épi. En ce qui concerne la productivité de ces épis, les symptômes observés sur tige n'ont eu de répercussion qu'une seule année (1983-1984) entraînant une diminution de $12 \%$ du poids de grains formés par épi chez la variété Roazon. Bien que les surfaces nécrosées sur tiges aient été relativement faibles cette année-là (moins de $25 \%$ ), les mêmes effets n'ont pas été observés les années suivantes avec des surfaces nécrosées supérieures à $25 \%$ laissant supposer que les conditions climatiques lors de la formation des grains jouent également un grand rôle dans l'accentuation de l'effet du parasite sur le rendement final.

Même s'il peut occasionnellement entraîner des chutes de rendement, le rhizoctone doit être considéré comme une maladie de faible nuisibilité. Cette observation rejoint les résultats obtenus par Clarkson et Cook (1983) en GrandeBretagne et par Meunier (1984) en Belgique. II est néanmoins important de souligner les dégâts observés aux stades jeunes de la culture par des attaques précoces et décrits ici pour la première fois. Si ces attaques sont rarement signalées, en conditions naturelles d'infestation dans la moitié nord de la France, elles sont plus fréquentes dans des conditions plus méridionales comme la vallée du Rhône par exemple.

De plus, cette faible nuisibilité ne doit pas nous faire oublier que le rhizoctone est une maladie de plus en plus fréquemment observée. Nous savons (Lucas et Cavelier, 1983) qu'il existe une grande variabilité dans l'espèce $R$ cerealis. Le maintien du champignon en phase parasitaire, conséquence des traitements phytosanitaires contre le piétin verse, peut laisser craindre une évolution des populations vers des souches de $R$ cerealis ayant des aptitudes parasitaires et une nuisibilité plus importantes.

Enfin, les attaques de rhizoctone n'étant que très rarement les seules attaques du pied dans 
un peuplement de blé d'hiver, il serait intéressant, dans une étude ultérieure, de prendre en compte la nuisibilité des complexes parasitaires ainsi constitués.

\section{REMERCIEMENTS}

Ce travail a été réalisé dans le cadre du contrat établi entre I'INRA et le GIE Club des 5.

\section{RÉFÉRENCES}

Boerema GH, Verhoeven AA (1977) Check list for scientific names of common parasitic fungi. Ser $2 \mathrm{~b}$ : Fungi on field crops: cereals and grasses. Neth $J$ Plant Pathol 83, 164-204

Cavelier N, Lucas P, Boulch G (1985) Évolution du complexe parasitaire constitué par Rhizoctonia cerealis Van der Hoeven et Pseudocercosporella herpotrichoides (Fron) Deighton, champignons parasites de la base des tiges de céréales. Agronomie 5, 693-700

Clarkson JDS, Cook RJ (1983) Effect of sharp eyespot (Rhizoctonia cerealis) on yield loss in winter wheat. Plant Pathol 32, 421-428

Doussinault G (1970) Problèmes posés par l'amélioration de la résistance du blé tendre vis-à-vis du piétin verse (Cercosporella herpotrichoides Fron). Ann Amélior Plant 20, 433-452

Doussinault G (1973) Comportement de 12 variétés de blé tendre vis-à-vis du piétin verse (Cercosporella herpotrichoides). Conséquences pour la sélection. Ann Amélior Plant 23, 333-346

Foex $E$ (1941) Un champignon à sclérote du blé. Son action parasitaire. Ann Epiphyt 7, 21-42

Glynne MD, Richtie WM (1943) Sharp eyespot of wheat caused by Corticium (Rhizoctonia) solani. Nature 152, 161
Hollins TW, Jellis GT, Scott PR (1983) Infection of potato and wheat isolates of Rhizoctonia solani and $R$ cerealis. Plant Pathol 32, 303-310

Lipps PE, Herr LJ (1982) Etiology of Rhizoctonia cerealis in sharp eyespot of wheat. Phytopathology 72, 1574-1577

Lucas P, Cavelier N (1983) Rhizoctonia cerealis Van der Hoeven, agent du rhizoctone des céréales. $\mathrm{Ca}$ ractéristiques et variabilité. Agronomie 3, 831-838

Meunier S (1984) Inventaire des maladies du pied chez le froment d'hiver en 1983 et incidence sur les composantes du rendement. Parasitica 40, 27-40

Oort AJP (1936) De oogvlekkenziekte van de granen, veroorzaakt door Cercosporella herpotrichoides Fron. Tijdschr Plantenziekten 42, 179

Pitt D (1964) Studies on sharp eyespot disease of cereals. I. Disease symptoms and pathogenicity of isolates of Rhizoctonia solani Kuhn and the influence of soil factors and temperature on disease development. Ann App/ Biol 54, 77-79

Pitt D (1966) Studies on sharp eyespot disease of cereals. II. Effects of the disease on the wheat host and the incidence of disease in the field. Ann Appl Biol 58, 299-308

Ponchet J (1959) La maladie du piétin verse des céréales : Cercosporella herpotrichoides Fron. Importance agronomique, biologie, épiphytologie. Ann Epiphyt (Paris) 10, 46-99

Rapilly F, Lemaire JM, Cassini R (1973) Les maladies des céréales. ITCF, Paris, $189 \mathrm{p}$

Reinecke P, Fehrmann F (1979) Rhizoctonia cerealis Van der Hoeven an Getreide in der Bundesrepublik Deutschland. Z Pflanzenkr Pflanzenschutz 86, 241246

Sprague R (1937) A further note on the fungus causing a white foot rot of wheat on oats. Phytopathology $27,798-800$

Zadoks JC, Chang TT, Konzak CF (1974) A decimal code for the growth stage of cereals. Weed Res 14 , 415-421 